\title{
Neutrino footprint in Large Scale Structure
}

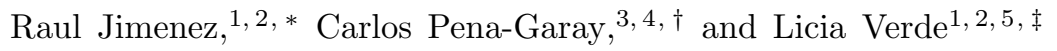 \\ ${ }^{1}$ ICREA \& ICC, University of Barcelona, Marti i Franques 1, Barcelona 08028, Spain. \\ ${ }^{2}$ Radcliffe Institute for Advanced Study, Harvard University, MA 02138, USA \\ ${ }^{3}$ Instituto de Fisica Corpuscular, CSIC-UVEG, P.O. 22085, Valencia, 46071, Spain \\ ${ }^{4}$ Laboratorio Subterráneo de Canfranc, Estación de Canfranc, 22880, Spain \\ ${ }^{5}$ Institute of Theoretical Astrophysics, University of Oslo, Oslo 0315, Norway.
}

\begin{abstract}
Recent constrains on the sum of neutrino masses inferred by analyzing cosmological data, show that detecting a non-zero neutrino mass is within reach of forthcoming cosmological surveys, implying a direct determination of the absolute neutrino mass scale. The measurement relies on constraining the shape of the matter power spectrum below the neutrino free streaming scale: massive neutrinos erase power at these scales. Detection of a lack of small-scale power, however, could also be due to a host of other effects. It is therefore of paramount importance to validate neutrinos as the source of power suppression at small scales. We show that, independent on hierarchy, neutrinos always show a footprint on large, linear scales; the exact location and properties can be related to the measured power suppression (an astrophysical measurement) and atmospheric neutrinos mass splitting (a neutrino oscillation experiment measurement). This feature can not be easily mimicked by systematic uncertainties or modifications in the cosmological model. The measurement of such a feature, up to $1 \%$ relative change in the power spectrum, is a smoking gun for confirming the determination of the absolute neutrino mass scale from cosmological observations. It also demonstrates the synergy of astrophysics and particle physics experiments.
\end{abstract}

PACS numbers:

In the past few years, there has been an amazing progress in cosmology. An accurate cosmic microwave background (CMB) spectrum, both in temperature and polarization has been measured by Planck [1] and WMAP [2]. The expansion history of the Universe has been mapped in several ways: with measurements of the Baryon Acoustic Oscillation (BAO) scale by the Baryon Oscillation Spectroscopic Survey (BOSS) of the Sloan Digital Sky Survey (SDSS) 3 and others 4, 5]; by the luminosity distance relation as given by Type $1 \mathrm{~A}$ supernova data e.g., [6]; via the direct measurement of the Hubble parameter with cosmic chronometers [7, 8].

Finally, large scale structure (LSS) has been probed by a variety of surveys (galaxies e.g., 9 12, weak lensing e.g., 13 17, Ly $\alpha$ [18) with increased sensitivity to the scale and redshift dependences of the matter power spectrum, thanks also to redshift space distortion measurements e.g., [19, 20].

All this wealth of cosmological data show a consistent $\Lambda$ CDM model with improved precision on parameters and better control of systematics. If included as a parameter in the model, total neutrino mass bounds have significantly improved in a variety of analysis, yielding an upper bound slightly higher than $100 \mathrm{meV}$ [18, 21]. Massive neutrinos free stream out of potential wells, erasing fluctuations and thus suppressing power on small scales e.g., [22, 23]; the measured small scale power is consistent with the standard (massless neutrino) $\Lambda$ CDM model and inconsistent with large neutrino masses. These bounds are very close to the limit that separates inverted and normal ordering and is within a factor of two of the lower limit of the sum of neutrino masses set by oscillations e.g., 24]. Near future progress in this measurement has an important impact on a crucial question in neutrino physics 25]: is neutrino its own anti-particle? The almost century-old question can be resolved if a neutrinoless double beta decay is observed [26, 27]. If light neutrinos are the main source of this decay, the measurement of the neutrino mass, provides an estimate of the half-live and therefore the size and level of background of experiments required to prove it.

Relevant cosmological constraints on neutrino masses (i.e., coming from measurements of the small scales power suppression) have to be matched with careful verification of systematic effects or alternative explanations of the data. Building on the work of [25], we show that cosmological data contain independent information on the neutrino masses that can be hardly mimicked by other effects. Driven by the current strong limits in the total mass, we demonstrate that there is sensitivity to measure the expected large mass splitting of neutrinos if the total mass is measured from forthcoming cosmological data. The agreement of the mass splitting inferred from cosmology and the large neutrino mass splitting measured by oscillation experiments should prove as convincing evidence of the discovery of relic neutrinos in cosmological surveys.

Massive neutrinos affect cosmological observations in a variety of different ways. For example, the combination of $\mathrm{CMB}$ and $\mathrm{BAO}$ data constrain the total neutrino mass $\Sigma<0.23 \mathrm{eV}$ at the $95 \%$ confidence level [1. Neutrinos with mass $\lesssim 1 \mathrm{eV}$ become non-relativistic after the epoch of recombination probed by the CMB, thus massive neutrinos alter matter-radiation equality for 


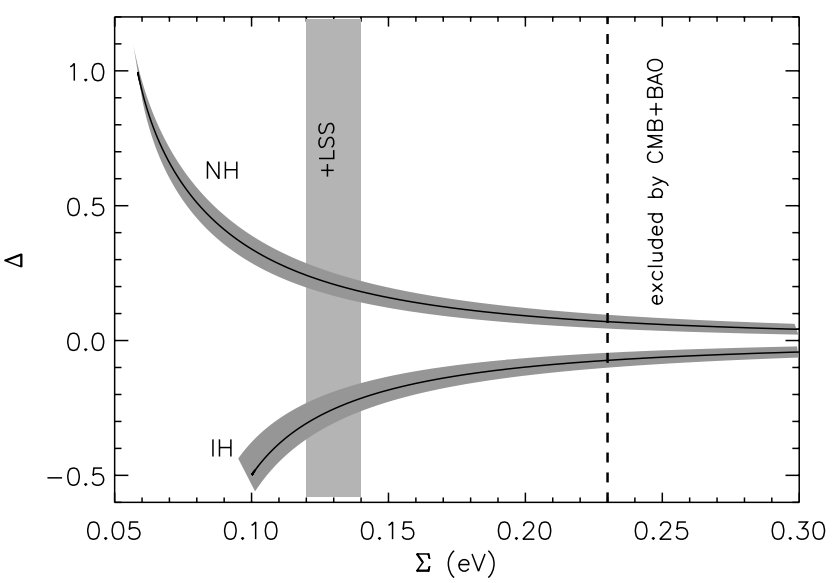

FIG. 1: Constraints from neutrino oscillations (shaded regions) and from cosmology in the $\Sigma-\Delta$ plane. In this parameterization the sign of $\Delta$ specifies the hierarchy.

a fixed $\Omega_{m} h^{2}$. After neutrinos become non-relativistic, their free streaming damps the small-scale power and modifies the shape of the matter power spectrum below the free-streaming length. Combining large-scale structure and CMB data, at present the sum of masses is constrained to be $\Sigma \lesssim 0.13 \mathrm{eV}$ [18, 21]. Forthcoming large-scale structure data promise to determine the small-scale $(0.1 \lesssim k \lesssim 1 \mathrm{~h} / \mathrm{Mpc})$ matter power spectrum exquisitely well and to yield errors on $\Sigma$ well below 0.1 eV (e.g., [28, 29]). Here, we assume the standard $\Lambda$ CDM model (plus massive neutrinos) and explore the changes in the matter power spectra due to the neutrino properties (mass and hierarchy).

Neutrino oscillation data have measured the neutrino squared mass differences, which are hierarchical. Given the smallness of neutrino masses and the hierarchy in mass splittings, we can characterize the impact of neutrino masses on cosmological observables and in particular on the matter power spectrum by two parameters: the total mass $\Sigma$ and the ratio of the largest mass splitting to the total mass, $\Delta$; while one can safely neglect the impact of the solar mass splitting in cosmology. In this excellent approximation, two masses characterize the neutrino mass spectrum, the lightest one, $m$, and the heaviest one, $M$.

We define the relation between the neutrino masses $m$ and $M$ and the parameters $\Sigma$ and $\Delta$ as

$$
\begin{array}{rll}
\mathrm{NH}: & \Sigma=2 m+M & \Delta=(M-m) / \Sigma \\
\mathrm{IH}: & \Sigma=m+2 M & \Delta=(m-M) / \Sigma .
\end{array}
$$

In Fig 1 we show the current constraints on neutrino mass properties in the $\Sigma-\Delta$ plane. We use the $\Delta$ param- eterization for the following reasons: $\Delta$ changes continuously through normal, degenerate and inverted hierarchies; $\Delta$ is positive for $\mathrm{NH}$ and negative for $\mathrm{IH}$; cosmological data are sensitive to $\Delta$ in an easily understood way through the largest mass splitting (i.e., the absolute value of $\Delta$ ), while the direction of the splitting (the sign of $\Delta$ ) introduces a sub-dominant correction to the main effect 25]. This parameterisation is strictly only applicable for $\Sigma>0$, but oscillations experiments already set $\Sigma>M \gtrsim 0.057 \mathrm{eV}$.

It is important to note that not the entire parameter space in the $\Sigma-\Delta$ plane (or of any other parameterization of the hierarchy used in the literature) is allowed by particle physics constraints: only the regions around the normal and inverted hierarchies allowed by neutrino oscillation experiments are physical (see Fig 1).

The effect of neutrino mass on the CMB is related to the physical density of neutrinos, and therefore the mass difference between eigenstates can be neglected. However individual neutrino masses can have an effect on the large-scale shape of the matter power spectrum. In fact, neutrinos of different masses have different transition redshifts from relativistic to non-relativistic behavior, and their individual masses and their mass splitting change the details of the radiation-domination to matterdomination regime. As a result, the detailed shape of the matter power spectrum on scales $k \lesssim 0.01 h / \mathrm{Mpc}$ is affected. Therefore a precise measurement of the matter power spectrum shape can give information on both the sum of the masses and the neutrino mass splitting.

To gain physical intuition on the effect of neutrino properties on cosmological observables, such as the shape of the matter power spectrum, it is useful to adopt the following analytical approximation, as described in Ref. [30. The matter power spectrum can be written as:

$$
\frac{k^{3} P(k ; z)}{2 \pi^{2}}=\Delta_{R}^{2} \frac{2 k^{2}}{5 H_{0}^{2} \Omega_{m}^{2}} D_{\nu}^{2}(k, z) T^{2}(k)\left(\frac{k}{k_{p}}\right)^{\left(n_{s}-1\right)},
$$

where $\Delta_{R}^{2}$ is the primordial amplitude of the fluctuations (evaluated at the pivot scale $k_{p}$ ), $n_{s}$ is the primordial power spectrum spectral slope, $T(k)$ denotes the matter transfer function and $D_{\nu}(k, z)$ is the scale-dependent linear growth function, which encloses the dependence of $P(k)$ on non-relativistic neutrino species.

Each of the three neutrinos contributes to the neutrino mass fraction $f_{\nu, i}$ where $i$ runs from 1 to 3 ,

$$
f_{\nu, i}=\frac{\Omega_{\nu, i}}{\Omega_{m}}=f_{0} \times m_{\nu_{i}}=0.01\left(\frac{m_{\nu_{i}}}{0.13 \mathrm{eV}}\right)\left(\frac{0.14}{\Omega_{m} h^{2}}\right)
$$

and has a free-streaming scale $k_{\mathrm{fs}, i}$,

$$
k_{\mathrm{fs}, i}=k_{0} \sqrt{m_{\nu_{i}}}=0.015 \sqrt{\frac{m_{\nu_{i}}}{0.13 \mathrm{eV}} \frac{\Omega_{m} h^{2}}{0.14} \frac{5}{1+z}} \mathrm{Mpc}^{-1} .
$$




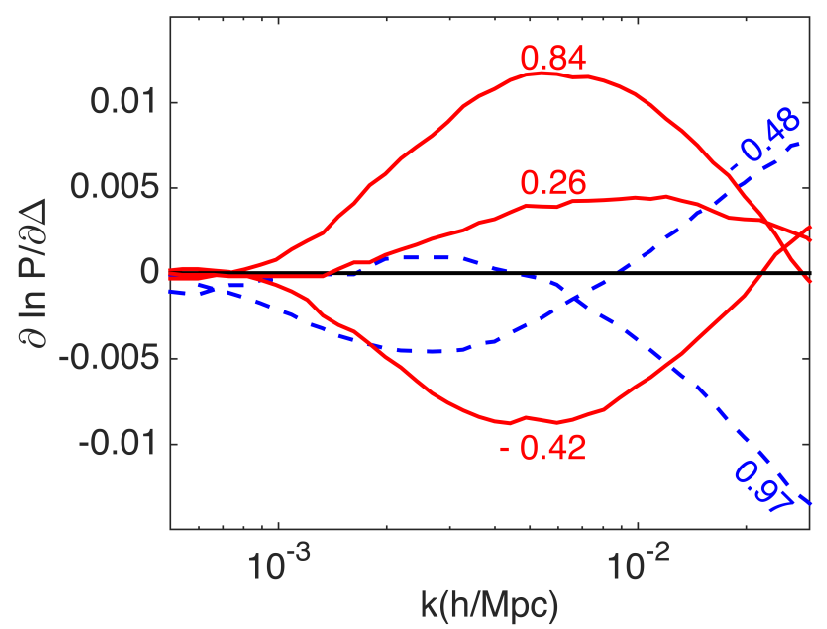

FIG. 2: Dependence of $P(k)$ on the parameter $\Delta$ at $z=0$, for fixed $\Sigma, 0.1 \mathrm{eV}$ (red full) and $0.06 \mathrm{eV}$ (blue dashed), and several values of $\Delta$. The dependence is expressed as fractional variation in $P(k)$ for a unit variation in $\Delta$. For $\Sigma=0.1 \mathrm{eV}$, normal (inverted) hierarchy from oscillation data correspond to $\Delta \sim 0.3(-0.5)$, as shown in Fig.1. The changes visible at $k>10^{-2} \mathrm{~h} / \mathrm{Mpc}$ for $\Sigma=0.06 \mathrm{eV}$ are due to $\Delta$ changing the matter-radiation equality and thus the shape parameter, being all other cosmological parameters kept fixed.

Analogously, one can define the corresponding quantities for the combined effect of all species, by using $\Sigma$ instead of $m_{\nu_{i}}$. Since we will only distinguish between a light and a heavy eigenstate we will have e.g., $f_{\nu, m}, f_{\nu, \Sigma}, k_{\mathrm{fs}, m}, k_{\mathrm{fs}, \Sigma}$ etc., where in the expression for $f_{\nu, m}$ one should use the mass of the eigenstate (which is the mass of the individual neutrino, or twice as much depending on the hierarchy) while in $k_{\mathrm{fs}, m}$ one should use the mass of the individual neutrino.

The dependence of $P(k)$ on non-relativistic neutrino species is in $D_{\nu}(k, z)$, given by

$$
D_{\nu_{i}}(k, z) \propto\left(1-f_{\nu_{i}}\right) D(z)^{1-p_{i}}
$$

where $k \gg k_{\mathrm{fs}, i}(z)$ and $p_{i}=\left(5-\sqrt{25-24 f_{\nu_{i}}}\right) / 4$. The standard linear growth function $D(z)$ fitting formula is taken from [31. $\Delta$ directly changes the growth on scales $k_{\mathrm{fs}, m}<k<k_{\mathrm{fs}, \Sigma}$, i.e., $\sqrt{(1-\Delta) / 3}<k /\left(k_{0} \sqrt{\Sigma}\right)<1$, which leads to changes in the power spectrum that can be aproximated at linear order by

$$
\frac{P(k ; z)_{\nu}-P(k ; z)}{P(k ; z)} \propto-f_{\nu, \Sigma}(1-\Delta) / 3 .
$$

Firstly, $\Sigma$ and $\Delta$ modify the range of scales where the impact of $\Delta$ is important. In particular, smaller total

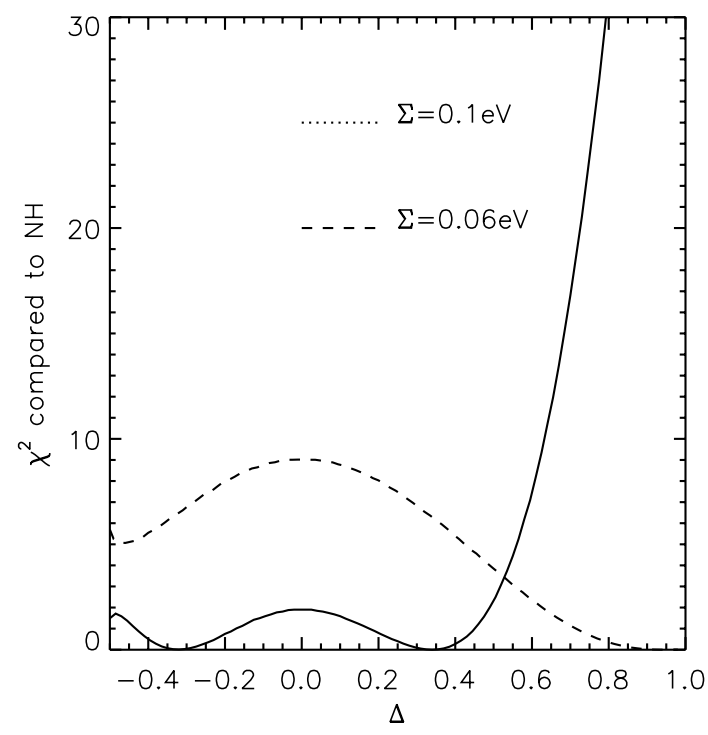

FIG. 3: Statistical power of future surveys, described in 25], to determine $\Delta$ for fixed values of $\Sigma$. We assume normal hierarchy fiducial model, with $\Delta$ from neutrinos oscillation data, Many $\Delta$ values potentially inferred from cosmological data can be statistically distinguished from the fiducial model.

mass of neutrino leads to a narrower range of larger scales where $\Delta$ influences the power spectrum. Secondly, while $\Delta=0$ leads to lack of a signature, positive (negative) $\Delta$ increases (decreases) linearly the amount of power at the scales of influence.

This description is, however, incomplete: the transition between the different regimes is done sharply in $k$ while in reality the change is very smooth. In addition, the individual masses change the details of the matterradiation transition which (keeping all other parameters fixed) adds an additional effect at scales $k>k_{\mathrm{fs}, \Sigma}$. In what follows we will therefore use the full numerical evaluation [32].

Throughout this paper we assume a fiducial model given by basic parameters of the standard LCDM cosmology and the fiducial values for $\Sigma$ and $\Delta$ are then further specified in each case. We have used the publicly available CAMB code 32 to numerically compute the matter power spectrum. In Fig. 2 we show the dependence of $P(k)$ on the parameter $\Delta$ at $z=0$ for fixed $\Sigma$ and fixed cosmological parameters. The dependence is shown as the fractional change of the matter power spectrum for a unit change of the parameter $\Delta$. In order to compute reliably the above derivatives, CAMB needs to be run at the highest precision settings, with fine $k$ sampling and taking care that interpolations procedures in-built in the code do not introduce a spurious signal. Note that 
the effect appears on large, linear scales. Moreover, the location, trend and amplitude of the maximum deviation of $\partial \ln P / \partial \Delta$ depend on $\Sigma$ : oscillation experiments constraints on mass splitting together with a value of $\Sigma$ completely specify the expected signal. In Fig. 3 we estimate the expected signal to noise in terms of difference in $\chi^{2}=-2 \ln L$ as a function of $\Delta$ fixing all other cosmological parameters, for a survey with the same characteristics as considered in 25. In this calculation we considered only scales $k<k_{\max }^{*}$ where $k_{\max }^{*}$ represent the scale above which $\partial \ln P / \partial \Delta$ becomes constant (and non-zero): by fixing all cosmological parameters, $\Delta$ alters matter radiation equality and so the $\Gamma$ shape parameter and the $P(k)$ normalisation at scales below equality, these changes would be canceled through cosmological degeneracies with other parameters (e.g., for $\Sigma=0.1 \mathrm{eV}$ $\left.k_{\max }^{*}=2.5 \times 10^{-2} \mathrm{~h} / \mathrm{Mpc}\right)$.

In other words, if for example the small scale power suppression signal indicates a $\Sigma=0.1(0.06) \mathrm{eV}$, the large scale measurement should yield a value consistent with $|\Delta| \sim 0.3(\Delta \sim 1)$. Excluding other values such as $\Delta=1$ $(\Delta=0$ or $\Delta<0)$, which, as Fig. 3 shows, future surveys have the statistical power for achieve, would offer a powerful consistency check on the $\Sigma$ determination. Conversely, excluding the expected value for $\Delta$ at high confidence would cast doubts on the interpretation of the data in terms of neutrino masses and standard neutrino properties 33 .

To summarize, we have shown the existence of a well defined footprint of neutrinos in the matter power spectrum if the total mass of neutrinos is measured in the upcoming cosmological data. This footprint is localised on large, linear scales, where systematic effects that plague the $\Sigma$-sensitive small mildly non-linear scales (non-linearities, non-linear bias, baryonic and astrophysical effects, shot noise etc.) are not present: the two signatures are not independent (in the standard model they are both made by neutrinos) but, because of the separation of scales, statistically uncorrelated. They could be partially correlated through cosmological parameter degeneracies but this is left for future work. The systematic effects at play on these large scales (i.e., modelling of the survey window function, selection function, photometric redshifts errors, if applicable, general relativistic effects, primordial-non-gaussianity) are completely independent form those acting at small scales and more benign, moreover they show a dependence on k-scale fundamentally different from the signal. One may wonder on the implications of this result. For example, not finding the power suppression should erase the footprint we discussed here, but will lead to modifications of either the cosmological model or the neutrino properties. Understanding the new physics beyond the standard (cosmology or neutrino) model would be within reach with improvements in neutrino beta and double-beta decay experiments and further cosmological surveys. Therefore a detection of this large-scale signature or a null result will have profound implications in neutrino physics and cosmology. A detection will be a "smoking gun" for verifying power suppression of small scales by neutrinos. As such it will be an indirect discovery of relic neutrinos and lead to the stronger bound on properties of neutrino like neutrino invisible decays, limiting very small neutrino couplings to scalars $\mathrm{g} \lesssim 4 \cdot 10^{-14}[34$.

Funding for this work was partially provided by the Spanish MINECO under projects FPA2014-57816P, AYA2014-58747-P and MDM-2014- 0369 of ICCUB (Unidad de Excelencia Maria de Maeztu), by Generalitat Valencia under Prometeo Grant II/2014/050 and by PITN- GA-2011-289442-INVISIBLES.

* Electronic address: raul.jimenez@icc.ub.edu

† Electronic address: penya@ific.uv.es

¥ Electronic address: liciaverde@icc.ub.edu

[1] P. A. R. Ade et al. [Planck Collaboration], arXiv:1502.01589 [astro-ph.CO]].

[2] G. Hinshaw et al. [WMAP Collaboration], Astrophys. J. Suppl. 208, 19 (2013) doi:10.1088/0067-0049/208/2/19 arXiv:1212.5226 [astro-ph.CO]].

[3] L. Anderson et al. [BOSS Collaboration], Mon. Not. Roy. Astron. Soc. 441, no. 1, 24 (2014) doi:10.1093/mnras/stu523 arXiv:1312.4877 [astroph.CO]].

[4] C. Blake et al., Mon. Not. Roy. Astron. Soc. 418, 1707 (2011) doi:10.1111/j.1365-2966.2011.19592.x arXiv:1108.2635 [astro-ph.CO]].

[5] F. Beutler et al., Mon. Not. Roy. Astron. Soc. 416, 3017 (2011) doi:10.1111/j.1365-2966.2011.19250.x arXiv:1106.3366 [astro-ph.CO]].

[6] M. Betoule et al. [SDSS Collaboration], Astron. Astrophys. 568 (2014) A22 doi:10.1051/0004-6361/201423413 arXiv:1401.4064 [astro-ph.CO]].

[7] M. Moresco et al., JCAP 1208 (2012) 006 doi:10.1088/1475-7516/2012/08/006 arXiv:1201.3609 [astro-ph.CO]].

[8] M. Moresco et al., arXiv:1601.01701 [astro-ph.CO].

[9] D. Parkinson et al., Phys. Rev. D 86 (2012) 103518 doi:10.1103/PhysRevD.86.103518 arXiv:1210.2130 [astro-ph.CO]].

[10] S. Riemer-Srensen, D. Parkinson and T. M. Davis, Phys. Rev. D 89 (2014) 103505 doi:10.1103/PhysRevD.89.103505 arXiv:1306.4153 [astro-ph.CO]].

[11] B. A. Reid et al., Mon. Not. Roy. Astron. Soc. 404 (2010) 60 doi:10.1111/j.1365-2966.2010.16276.x arXiv:0907.1659 [astro-ph.CO]].

[12] S. Alam et al. [SDSS-III Collaboration], Astrophys. J. Suppl. 219 (2015) 1, 12 doi:10.1088/0067-0049/219/1/12 arXiv:1501.00963 [astro-ph.IM]].

[13] M. J. Jee, J. A. Tyson, S. Hilbert, M. D. Schneider, S. Schmidt and D. Wittman, arXiv:1510.03962 [astroph.CO].

[14] L. Van Waerbeke et al., Mon. Not. Roy. Astron. Soc. 433 (2013) 3373 doi:10.1093/mnras/stt971 arXiv:1303.1806 
[astro-ph.CO]].

[15] M. Kilbinger et al., Mon. Not. Roy. Astron. Soc. 430 (2013) 2200 doi:10.1093/mnras/stt041 arXiv:1212.3338 [astro-ph.CO]].

[16] T. D. Kitching et al. [CFHTLenS Collaboration], Mon. Not. Roy. Astron. Soc. 442 (2014) 2, 1326 doi:10.1093/mnras/stu934 arXiv:1401.6842 [astroph.CO]].

[17] T. Abbott et al. [DES Collaboration], arXiv:1507.05552 [astro-ph.CO].

[18] N. Palanque-Delabrouille et al., JCAP 1511, no. $11, \quad 011$ (2015) doi:10.1088/1475-7516/2015/11/011 arXiv:1506.05976 [astro-ph.CO]].

[19] L. Samushia et al., Mon. Not. Roy. Astron. Soc. 439 (2014) 4, 3504 doi:10.1093/mnras/stu197 arXiv:1312.4899 [astro-ph.CO]].

[20] F. Beutler et al. [BOSS Collaboration], Mon. Not. Roy. Astron. Soc. 443 (2014) 2, 1065 doi:10.1093/mnras/stu1051 arXiv:1312.4611 [astroph.CO]].

[21] A. J. Cuesta, V. Niro and L. Verde, arXiv:1511.05983 [astro-ph.CO].

[22] W. Hu, D. J. Eisenstein and M. Tegmark, Phys. Rev. Lett. 80 (1998) 5255 doi:10.1103/PhysRevLett.80.5255 astro-ph/9712057.

[23] J. Lesgourgues and S. Pastor, Phys. Rept. 429 (2006) 307 doi:10.1016/j.physrep.2006.04.001 astro-ph/0603494.

[24] M. C. Gonzalez-Garcia, M. Maltoni and T. Schwetz, arXiv:1512.06856 [hep-ph].

[25] R. Jimenez, T. Kitching, C. Pena-Garay and L. Verde, JCAP 1005 (2010) 035 doi:10.1088/14757516/2010/05/035 arXiv:1003.5918 [astro-ph.CO]].

[26] W. H. Furry, Phys. Rev. 56, 1184 (1939). doi:10.1103/PhysRev.56.1184

[27] E. Majorana, Nuovo Cim. 14, 171 (1937). doi:10.1007/BF02961314

[28] C. Carbone, L. Verde, Y. Wang and A. Cimatti, JCAP 1103 (2011) 030 doi:10.1088/1475-7516/2011/03/030 arXiv:1012.2868 [astro-ph.CO]].

[29] B. Audren, J. Lesgourgues, S. Bird, M. G. Haehnelt and M. Viel, JCAP 1301 (2013) 026 doi:10.1088/14757516/2013/01/026 arXiv:1210.2194 [astro-ph.CO]].

[30] M. Takada, E. Komatsu and T. Futamase, Phys. Rev. D 73, 083520 (2006).

[31] D. J. Eisenstein and W. Hu, Astrophys. J. 511 (1997) 5.

[32] A. Lewis, A. Challinor and A. Lasenby, Astrophys. J. 538, 473 (2000) doi:10.1086/309179 astro-ph/9911177.

[33] R. Jimenez, C. Pena-Garay and L. Verde, in prep.

[34] P. D. Serpico, Phys. Rev. Lett. 98, 171301 (2007) doi:10.1103/PhysRevLett.98.171301 astro-ph/0701699. 